\title{
On effective actions of BPS branes and their higher derivative corrections
}

\author{
Ehsan Hatefi ${ }^{1}$ \\ Theory Group, Physics Department, CERN, \\ CH-1211, Geneva 23, Switzerland \\ E-mail: ehsan.hatefi@cern.ch
}

ABSTRACT: We calculate in detail the disk level S-matrix element of one Ramond-Ramond field and three gauge field vertex operators in the world volume of BPS branes, to find four gauge field couplings to all orders of $\alpha^{\prime}$ up to on-shell ambiguity. Then using these infinite couplings we find that the massless pole of the field theory amplitude is exactly equal to the massless pole S-matrix element of this amplitude for the $p=n$ case to all orders of $\alpha^{\prime}$. Finally we show that the infinite massless poles and the contact terms of this amplitude for the $p=n+2$ case can be reproduced by the Born-Infeld action and the Wess-Zumino actions, and by their higher derivative corrections.

Keywords: Conformal Field Models in String Theory, D-branes

ARXIV EPRINT: 1003.0314

\footnotetext{
${ }^{1}$ On leave of Ferdowsi University of Mashhad, School of Physics, Institute for research in fundamental sciences (IPM), P.O.Box 19395-5531, Tehran, Iran.
} 


\section{Contents}

1 Introduction 1

2 The four point superstring scattering (CAAA) 4

$\begin{array}{lll}3 & \text { Momentum expansion } & 7\end{array}$

4 Low energy field theory $\quad 9$

$\begin{array}{ll}4.1 p=n+4 \text { case } & 10\end{array}$

$\begin{array}{ll}4.2 p=n \text { case } & 10\end{array}$

$\begin{array}{lll}4.3 & \text { First massless pole for } p=n \text { case } & 11\end{array}$

5 Four gauge field couplings $\quad \mathbf{1 2}$

$\begin{array}{lll}5.1 & \text { Infinite massless poles for } p=n \text { case } & 13\end{array}$

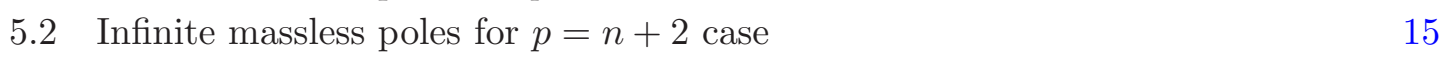

$\begin{array}{lll}5.3 \text { Contact terms } & 17\end{array}$

\section{Introduction}

A $\mathrm{D}_{p}$-brane is a $(p+1)$-dimensional hypersurface in a space-time defined such that open strings can end on it. They are sources of Ramond-Ramond $(\mathrm{P}+1)$-form fields in IIA and IIB string theories [1]. They have been studied and many properties of them have been investigated [2-4], being the center of attention both in theory and phenomenology. The stable $\mathrm{D}_{p}$-branes ( $p$ is even in IIA and odd in IIB theory) preserve half of supersymmetry. This implies that the spectrum of open strings has 16 supersymmetries and has to be tachyon-free. An important point is that the supersymmetry transformation requires the momenta of the scattering states to be only along the brane directions.

In fact, stability, supersymmetry, conserved Ramond-Ramond (RR) charge and having no tachyons are all properties of these type II $\mathrm{D}_{p}$-branes. All supersymmetric $\mathrm{D}_{p}$-branes in IIA can be generated as bound states of $\mathrm{D}_{9}$-branes [5]. They can also be derived from K-theory [6].

The world-volume theory of a $\mathrm{D}_{p}$-brane involves a massless $\mathrm{U}(1)$ vector $A_{a}, 9-p$ real massless scalars $\phi^{i}$ which describe transverse oscillations of the brane and their super partner fermions [7]. At leading order, the low-energy action for these fields corresponds to the dimensional reduction of a ten-dimensional U(1) super-Yang Mills theory. There are higher order $\alpha^{\prime}=l_{s}^{2}$ corrections, where $l_{s}$ is the string length scale. When derivatives of the field strengths (and second derivatives of the scalars) are small on the string scale, then the action to all orders in the field strength takes the Born-Infeld form [8-10] (also see [11]).

On the other hand, when there are $\mathrm{N}$ coincident $\mathrm{D}_{p}$-branes, the $\mathrm{U}(1)$ gauge symmetry of a single $\mathrm{D}_{p}$-brane is enhanced to the non-abelian $\mathrm{U}(N)$ symmetry [2]. The action for 
constructing non-abelian $\mathrm{D}_{p}$-branes on a general background was given by Myers [12] and other authors [13].

The low energy action describing the dynamics of $\mathrm{D}_{p}$-branes consists of two parts. The first part is Born-Infeld action

$$
S_{\mathrm{BI}}=-T_{p} \int d^{p+1} \sigma \mathrm{S} \operatorname{Tr}\left(e^{-\phi} \sqrt{-\operatorname{det}\left(P\left[E_{a b}+E_{a i}\left(Q^{-1}-\delta\right)^{i j} E_{j b}\right]+\lambda F_{a b}\right) \operatorname{det}\left(Q^{i} j\right)}\right),
$$

with

$$
E_{a b}=G_{a b}+B_{a b} \quad, \quad Q^{i}{ }_{j} \equiv \delta^{i}{ }_{j}+i \lambda\left[\Phi^{i}, \Phi^{k}\right] E_{k j}
$$

where $\lambda=2 \pi \ell_{s}^{2}, T_{p}$ is the brane tension, $\mathrm{P}[\ldots]$ indicates pull-back of background metric and NSNS two-form $(\mathrm{a}, \mathrm{b}=0, \ldots, 9), F_{a b}$ is the field strength of gauge field and $\mathrm{S} \operatorname{Tr}(\ldots)$ is symmetric trace prescription. For more details see [12]. In addition BI action provides the kinetic terms for the world-volume fields, it also contains the couplings of the $\mathrm{D}_{p}$-brane to the massless Neveu-Schwarz fields in the bulk. The second part is the Wess-Zumino action, which contains the coupling of the $\mathrm{U}(N)$ massless world volume vectors to the closed string RR field (indicated by $C$ ) $[1,14-16]$

$$
S_{\mathrm{WZ}}=\mu_{p} \int_{\Sigma_{(p+1)}} C \wedge \operatorname{Tr} e^{i 2 \pi \alpha^{\prime} F} .
$$

where $\Sigma_{(p+1)}$ is the world volume, Tr is over the Chan-Paton factors, $\mu_{p}$ is the RR charge of branes and $F$ is the field strength of the gauge field which defined $F=\frac{1}{2} F_{a b} d x^{a} \wedge d x^{b}$. Using the Taylor expansion one finds various couplings including two gauge fields, three gauge fields and so on. Therefore the effective theory has two parts

$$
S_{\mathrm{BPS}}=S_{\mathrm{BI}}+S_{\mathrm{WZ}}
$$

One method for finding these effective actions is the BSFT. By applying this formalism we can find WZ couplings. In this framework, it has been argued in [17] when the RR field is constant, there is no higher derivative correction to the WZ couplings. The WZ term in this formalism is given by $[17,18]$

$$
S_{\mathrm{WZ}}=\mu_{p}^{\prime} \int_{\Sigma_{(p+1)}} C \wedge \operatorname{Str} e^{i 2 \pi \alpha^{\prime} \mathcal{F}},
$$

in which $\mathcal{F}$ is the curvature of superconnection. To study WZ couplings for BPS branes we use the second approach, which is the S-matrix method. As we are working with BornInfeld action in a flat background, we set $G_{a b}=\eta_{a b}$ and $B_{a b}=0$. The Born-Infeld action can be written as

$$
S_{\mathrm{BI}}=-T_{p} \int d^{p+1} \sigma \mathrm{S} \operatorname{Tr}\left(e^{-\phi} \sqrt{-\operatorname{det}\left(\eta_{a b}+2 \pi \alpha^{\prime} F_{a b}\right)}\right) .
$$

One important tool in string theory is scattering theory. In fact, the string theory corrections to field theory may be found perturbatively in $\alpha^{\prime}$ by means of scattering amplitude arguments. In string theory, one can narrate the scattering of closed strings from a $\mathrm{D}_{p}$-brane as follows : 
The string background is taken to be flat space, however, interactions of closed strings with a $\mathrm{D}_{p}$-brane are described by world-sheets with boundary. The boundary of world sheets must be fixed to the surface at the position of the $\mathrm{D}_{p}$-brane. In fact, we must consider Dirichlet boundary conditions on the fields transverse to the $\mathrm{D}_{p}$-brane and Neumann boundary conditions on the fields along world volume of the $\mathrm{D}_{p}$-brane $[19,20]$. The appearence of the $\mathrm{D}_{p}$-brane physics has yielded a deep change in the significance of open strings, with some efforts for explaining them being related to [21, 22]. It was conjectured that quantum effects of open strings moving on $\mathrm{D}_{p}$-branes will produce $\mathrm{D}_{p}$-branes geometry. Some previous works on scattering that involved a $\mathrm{D}_{p}$-brane and some works about applications on $\mathrm{D}_{p}$-branes can be found in [23-37]. Firstly the massless vertex operators were constructed for the external open strings on the $\mathrm{D}_{p}$-branes. For the scattering of massless states the $\mathrm{D}_{p}$-brane geometry is the extremal case. One can calculate the scattering of massless states from supersymmetric $\mathrm{D}_{p}$-branes in type II theory.

T-duality transformation transforms $\mathrm{D}_{p}$-branes from type IIA to type IIB. Using Tduality transformation one can substitute a scalar field with a gauge field and vice versa. Open string states on the $\mathrm{D}_{p}$-brane do not have transverse components of their momenta. In this paper, we would like to use T-duality to find higher derivative couplings of four gauge fields from higher derivative couplings of four transverse scalar fields [38].

The organization of the paper is as follows. In section 2 we calculate a tree-level four point string scattering including, one RR and three gauge field vertex operators in the world volume type II superstring theory and make a few remarks by studying direct computations. The world-volume theory can be described by Berkovits's superstring field theory [39]. Also the world-volume theory may be rewritten in terms of massless fields and an infinite number of their derivatives. In section 3 we examine the low energy limit by sending all Mandelstam variables to zero. In section 4 we consider low energy field theory to find desired couplings and then using these couplings we produce first massless pole for $p=n$ case.

To obtain the infinite massless poles for this case, one needs to know the higher derivative couplings of four gauge fields. To this aim, using T-duality transformation in section 5 we find higher derivative couplings of four gauge fields up to on-shell ambiguity. Then using these couplings in field theory we will show that the massless poles of this S-matrix element are exactly reproduced to all orders of $\alpha^{\prime}$. Finally in section 5.2 we obtain all infinite massless poles in field theory for $p=n+2$ case and find a consistent result between string theory and field theory amplitudes. In addition we generate contact terms of this amplitude in leading order and next to the leading order.

Before continuing our calculations, let us explain the conventions. Our index conventions are that lowercase Greek indices take values in the whole ten-dimensional spacetime, e.g., $\mu, \nu=0,1, \ldots, 9$; early Latin indices take values in the world-volume, e.g., $a, b, c=0$, $1, \ldots, \mathrm{p}$; and middle Latin indices take values in the transverse space, e.g., $i, j=\mathrm{p}+1, \ldots, 9$. Thus, for example, $G_{\mu \nu}$ indicates the entire spacetime metric, while $G_{a b}$ and $G_{i j}$ indicate metric components for directions parallel and orthogonal to the $\mathrm{D}_{p}$-branes, respectively. 


\section{The four point superstring scattering (CAAA)}

In this section, using the conformal field theory technique we evaluate the string scattering amplitude to find all couplings of one closed string $\mathrm{RR}$ field to three gauge fields on the world-volume of a single BPS $\mathrm{D}_{p}$-brane with flat empty space background. To calculate a S-matrix element, one firstly needs to choose the picture of the vertex operators. The sum of the superghost charges must be -2 for disk level amplitudes.

A great deal of effort for the scattering amplitudes at tree level has been made [40-44]. The S-matrix element of one closed string RR field and three gauge fields is given by the following correlation function

$$
\mathcal{A}^{\mathrm{CAAA}} \sim \int d x_{1} d x_{2} d x_{3} d z d \bar{z}\left\langle V_{A}^{(0)}\left(x_{1}\right) V_{A}^{(0)}\left(x_{2}\right) V_{A}^{(-1)}\left(x_{3}\right) V_{\mathrm{RR}}^{\left(-\frac{1}{2},-\frac{1}{2}\right)}(z, \bar{z})\right\rangle,
$$

where the closed string vertex operator inserted at the middle and open string vertex operators at the boundary of the disk world-sheet. The vertex operators in (2.1) are given as $^{1}$

$$
\begin{aligned}
V_{A}^{(0)}(x) & =\xi_{a}\left(\partial X^{a}(x)+2 i k \cdot \psi \psi^{a}(x)\right) e^{2 i k \cdot X(x)}, \\
V_{A}^{(-1)}(y) & =\xi \cdot \psi(y) e^{-\phi(y)} e^{2 i k \cdot X(y)}, \\
V_{\mathrm{RR}}^{\left(-\frac{1}{2},-\frac{1}{2}\right)}(z, \bar{z}) & =\left(P_{-} H_{(n)} M_{p}\right)^{\alpha \beta} e^{-\phi(z) / 2} S_{\alpha}(z) e^{i p \cdot X(z)} e^{-\phi(\bar{z}) / 2} S_{\beta}(\bar{z}) e^{i p \cdot D \cdot X(\bar{z})},
\end{aligned}
$$

where $k$ is the momentum of gauge field which satisfies the on-shell condition $k^{2}=0$ and $k . \xi=0$. The projector in the $\mathrm{RR}$ vertex operator is $P_{-}=\frac{1}{2}\left(1-\gamma^{11}\right)$ and

$$
H_{(n)}=\frac{a_{n}}{n !} H_{\mu_{1} \ldots \mu_{n}} \gamma^{\mu_{1}} \ldots \gamma^{\mu_{n}},
$$

where $n=2,4$ for type IIA and $n=1,3,5$ for type IIB. $a_{n}=i$ for IIA and $a_{n}=1$ for IIB theory. The spinorial indices are raised with the charge conjugation matrix, i.e., $\left(P_{-} H_{(n)}\right)^{\alpha \beta}=C^{\alpha \delta}\left(P_{-} H_{(n)}\right)_{\delta}{ }^{\beta}$ (for more conventions and notations see appendix B of [45]). The RR bosons are massless so $p^{2}=0$. The world-sheet fields have been extended to the entire complex plane. That is, we have replaced

$$
\tilde{X}^{\mu}(\bar{z}) \rightarrow D_{\nu}^{\mu} X^{\nu}(\bar{z}), \quad \tilde{\psi}^{\mu}(\bar{z}) \rightarrow D_{\nu}^{\mu} \psi^{\nu}(\bar{z}), \quad \tilde{\phi}(\bar{z}) \rightarrow \phi(\bar{z}), \quad \text { and } \quad \tilde{S}_{\alpha}(\bar{z}) \rightarrow M_{\alpha}{ }^{\beta} S_{\beta}(\bar{z}),
$$

where

$$
D=\left(\begin{array}{cc}
-1_{9-p} & 0 \\
0 & 1_{p+1}
\end{array}\right), \quad \text { and } \quad M_{p}=\left\{\begin{array}{cc}
\frac{ \pm i}{(p+1) !} \gamma^{a_{1}} \gamma^{a_{2}} \ldots \gamma^{a_{p+1}} \epsilon_{a_{1} \ldots a_{p+1}} & \text { for } p \text { even } \\
\frac{ \pm 1}{(p+1) !} \gamma^{a_{1}} \gamma^{a_{2}} \ldots \gamma^{a_{p+1}} \gamma_{11} \epsilon_{a_{1} \ldots a_{p+1}} & \text { for podd }
\end{array}\right.
$$

Using this doubling trick, one can find the standard holomorphic correlators for the worldsheet fields $X^{\mu}, \psi^{\mu}, \phi$ as the following

$$
\begin{aligned}
\left\langle X^{\mu}(z) X^{\nu}(w)\right\rangle & =-\eta^{\mu \nu} \log (z-w), \\
\left\langle\psi^{\mu}(z) \psi^{\nu}(w)\right\rangle & =-\eta^{\mu \nu}(z-w)^{-1}, \\
\langle\phi(z) \phi(w)\rangle & =-\log (z-w) .
\end{aligned}
$$

\footnotetext{
${ }^{1}$ In string theory, we set $\alpha^{\prime}=2$.
} 
Introducing $x_{4} \equiv z=x+i y$ and $x_{5} \equiv \bar{z}=x-i y$, the amplitude reduces to the following correlators for 123 ordering

$$
\begin{aligned}
\mathcal{A}^{\mathrm{CAAA}} \sim & \int d x_{1} d x_{2} d x_{3} d x_{4} d x_{5}\left(P_{-} H_{(n)} M_{p}\right)^{\alpha \beta} \xi_{1 a} \xi_{2 b} \xi_{3 c} x_{45}^{-1 / 4}\left(x_{34} x_{35}\right)^{-1 / 2} \\
& \times\left(I_{1}+I_{2}+I_{3}+I_{4}\right) \operatorname{Tr}\left(\lambda_{1} \lambda_{2} \lambda_{3}\right)
\end{aligned}
$$

where $x_{i j}=x_{i}-x_{j}$, with the Wick theorem one can find the correlators as

$$
\begin{aligned}
I_{1}= & <: \partial X^{a}\left(x_{1}\right) e^{2 i k_{1} \cdot X\left(x_{1}\right)}: \partial X^{b}\left(x_{2}\right) e^{2 i k_{2} \cdot X\left(x_{2}\right)}: e^{2 i k_{3} \cdot X\left(x_{3}\right)}: e^{i p . X\left(x_{4}\right)}: e^{i p . D \cdot X\left(x_{5}\right)}:> \\
& \times<: S_{\alpha}\left(x_{4}\right): S_{\beta}\left(x_{5}\right): \psi^{c}\left(x_{3}\right):> \\
I_{2}= & <: \partial X^{a}\left(x_{1}\right) e^{2 i k_{1} \cdot X\left(x_{1}\right)}: e^{2 i k_{2} \cdot X\left(x_{2}\right)}: e^{2 i k_{3} \cdot X\left(x_{3}\right)}: e^{i p \cdot X\left(x_{4}\right)}: e^{i p \cdot D \cdot X\left(x_{5}\right)}:> \\
& \times<: S_{\alpha}\left(x_{4}\right): S_{\beta}\left(x_{5}\right): 2 i k_{2} \cdot \psi \psi^{b}\left(x_{2}\right): \psi^{c}\left(x_{3}\right):> \\
I_{3}= & <: e^{2 i k_{1} \cdot X\left(x_{1}\right)}: \partial X^{b}\left(x_{2}\right) e^{2 i k_{2} \cdot X\left(x_{2}\right)}: e^{2 i k_{3} \cdot X\left(x_{3}\right)}: e^{i p . X\left(x_{4}\right)}: e^{i p . D \cdot X\left(x_{5}\right)}:> \\
& \times<: S_{\alpha}\left(x_{4}\right): S_{\beta}\left(x_{5}\right): 2 i k_{1} \cdot \psi \psi^{a}\left(x_{1}\right): \psi^{c}\left(x_{3}\right):> \\
I_{4}= & <: e^{2 i k_{1} \cdot X\left(x_{1}\right)}: e^{2 i k_{2} \cdot X\left(x_{2}\right)}: e^{2 i k_{3} \cdot X\left(x_{3}\right)}: e^{i p \cdot X\left(x_{4}\right)}: e^{i p \cdot D \cdot X\left(x_{5}\right)}:> \\
& \times<: S_{\alpha}\left(x_{4}\right): S_{\beta}\left(x_{5}\right): 2 i k_{1} \cdot \psi \psi^{a}\left(x_{1}\right): 2 i k_{2} \cdot \psi \psi^{b}\left(x_{2}\right): \psi^{c}\left(x_{3}\right):>.
\end{aligned}
$$

Using the first correlator, it is not difficult to calculate the correlators of $X$. We use the Wick-like rule [46] and [47] to find the correlation function involving an arbitrary number of world-sheet fermions $(\psi \mathrm{s})$ and two spin operators $(S S)$. One can generalize the Wicklike rule to find the correlation function of two spin operators and an arbitrary number of currents [43]. The only important point in using the Wick-like rule for currents is that one must not consider the Wick-like contraction for the two $\psi \mathrm{s}$ in one current. Taking this into account we can obtain the correlation function between two spin operators, one current and one world-sheet fermion as follows

$$
\begin{aligned}
I_{5}^{c b d}= & <: S_{\alpha}\left(x_{4}\right): S_{\beta}\left(x_{5}\right): \psi^{d} \psi^{b}\left(x_{2}\right): \psi^{c}\left(x_{3}\right):> \\
= & \left\{\left(\Gamma^{c b d} C^{-1}\right)_{\alpha \beta}+\frac{2 \operatorname{Re}\left[x_{24} x_{35}\right]}{x_{23} x_{45}}\left(\eta^{d c}\left(\gamma^{b} C^{-1}\right)_{\alpha \beta}-\eta^{b c}\left(\gamma^{d} C^{-1}\right)_{\alpha \beta}\right)\right\} \\
& \times 2^{-3 / 2} x_{45}^{1 / 4}\left(x_{24} x_{25}\right)^{-1}\left(x_{34} x_{35}\right)^{-1 / 2} .
\end{aligned}
$$

The calculation of the correlation function between two spin operators, two currents and one world-sheet fermion is more complicated, but using this generalization it is simply given by

$$
\begin{aligned}
I_{6}^{\text {cbeaf }}= & <: S_{\alpha}\left(x_{4}\right): S_{\beta}\left(x_{5}\right): \psi^{f} \psi^{a}\left(x_{1}\right): \psi^{e} \psi^{b}\left(x_{2}\right): \psi^{c}\left(x_{3}\right):> \\
= & \left\{\left(\Gamma^{c b e a f} C^{-1}\right)_{\alpha \beta}+2 r_{1} \frac{\operatorname{Re}\left[x_{14} x_{25}\right]}{x_{12} x_{45}}+2 r_{2} \frac{\operatorname{Re}\left[x_{14} x_{35}\right]}{x_{13} x_{45}}+2 r_{3} \frac{\operatorname{Re}\left[x_{24} x_{35}\right]}{x_{23} x_{45}}+4 r_{4}\right. \\
& \times\left(\frac{\operatorname{Re}\left[x_{14} x_{25}\right]}{x_{12} x_{45}}\right)^{2}+4 r_{5}\left(\frac{\operatorname{Re}\left[x_{14} x_{25}\right]}{x_{12} x_{45}} \times \frac{\operatorname{Re}\left[x_{14} x_{35}\right]}{x_{13} x_{45}}\right)+4 r_{6}\left(\frac{\operatorname{Re}\left[x_{14} x_{25}\right]}{x_{12} x_{45}}\right. \\
& \left.\left.\times \frac{\operatorname{Re}\left[x_{24} x_{35}\right]}{x_{23} x_{45}}\right)\right\} 2^{-5 / 2} x_{45}^{5 / 4}\left(x_{14} x_{15} x_{24} x_{25}\right)^{-1}\left(x_{34} x_{35}\right)^{-1 / 2}
\end{aligned}
$$


where

$$
\begin{aligned}
& r_{1}=\left(\eta^{f e}\left(\Gamma^{c b a} C^{-1}\right)_{\alpha \beta}-\eta^{f b}\left(\Gamma^{c e a} C^{-1}\right)_{\alpha \beta}-\eta^{a e}\left(\Gamma^{c b f} C^{-1}\right)_{\alpha \beta}+\eta^{a b}\left(\Gamma^{c e f} C^{-1}\right)_{\alpha \beta}\right), \\
& r_{2}=\left(\eta^{f c}\left(\Gamma^{b e a} C^{-1}\right)_{\alpha \beta}-\eta^{a c}\left(\Gamma^{b e f} C^{-1}\right)_{\alpha \beta}\right), \\
& r_{3}=\left(\eta^{e c}\left(\Gamma^{b a f} C^{-1}\right)_{\alpha \beta}-\eta^{b c}\left(\Gamma^{e a f} C^{-1}\right)_{\alpha \beta}\right), \\
& r_{4}=\left(\left(-\eta^{f e} \eta^{a b}+\eta^{f b} \eta^{a e}\right)\left(\gamma^{c} C^{-1}\right)_{\alpha \beta}\right), \\
& r_{5}=\left(\left(\eta^{f e} \eta^{a c}-\eta^{f c} \eta^{a e}\right)\left(\gamma^{b} C^{-1}\right)_{\alpha \beta}+\left(-\eta^{f b} \eta^{a c}+\eta^{f c} \eta^{a b}\right)\left(\gamma^{e} C^{-1}\right)_{\alpha \beta}\right), \\
& r_{6}=\left(\left(-\eta^{f e} \eta^{b c}+\eta^{f b} \eta^{e c}\right)\left(\gamma^{a} C^{-1}\right)_{\alpha \beta}+\left(\eta^{a e} \eta^{b c}-\eta^{a b} \eta^{e c}\right)\left(\gamma^{f} C^{-1}\right)_{\alpha \beta}\right) .
\end{aligned}
$$

Replacing the above spin correlators in (2.3) and performing the correlators over $X$, one finds:

$$
\begin{aligned}
\mathcal{A}^{\mathrm{CAAA}} \sim & \int d x_{1} d x_{2} d x_{3} d x_{4} d x_{5}\left(P_{-} H_{(n)} M_{p}\right)^{\alpha \beta} I \xi_{1 a} \xi_{2 b} \xi_{3 c} x_{45}^{-1 / 4}\left(x_{34} x_{35}\right)^{-1 / 2} \\
& \times\left(I_{7}^{c}\left(-\eta^{a b} x_{12}^{-2}+a_{1}^{a} a_{2}^{b}\right)+a_{1}^{a} a_{3}^{c b}+a_{2}^{b} a_{4}^{c a}-4 k_{1 f} k_{2 e} I_{6}^{c b e a f}\right) \operatorname{Tr}\left(\lambda_{1} \lambda_{2} \lambda_{3}\right),
\end{aligned}
$$

where $I_{6}^{\text {cbeaf }}$ is given in (2.5) and

$$
\begin{aligned}
I= & \left|x_{12}\right|^{4 k_{1} \cdot k_{2}}\left|x_{13}\right|^{4 k_{1} \cdot k_{3}}\left|x_{14} x_{15}\right|^{2 k_{1} \cdot p}\left|x_{23}\right|^{4 k_{2} \cdot k_{3}}\left|x_{24} x_{25}\right|^{2 k_{2} \cdot p}\left|x_{34} x_{35}\right|^{2 k_{3} \cdot p}\left|x_{45}\right|^{p . D \cdot p}, \\
a_{1}^{a}= & -i k_{2}^{a}\left(\frac{x_{42}}{x_{41} x_{12}}+\frac{x_{52}}{x_{51} x_{12}}\right)-i k_{3}^{a}\left(\frac{x_{43}}{x_{41} x_{13}}+\frac{x_{53}}{x_{51} x_{13}}\right), \\
a_{2}^{b}= & -i k_{1}^{b}\left(\frac{x_{14}}{x_{42} x_{12}}+\frac{x_{15}}{x_{52} x_{12}}\right)-i k_{3}^{b}\left(\frac{x_{43}}{x_{42} x_{23}}+\frac{x_{53}}{x_{52} x_{23}}\right), \\
a_{3}^{c b}= & 2 i k_{2 d} I_{5}^{c b d} \\
a_{4}^{c a}= & 2 i k_{1 e} 2^{-3 / 2} x_{45}^{1 / 4}\left(x_{14} x_{15}\right)^{-1}\left(x_{34} x_{35}\right)^{-1 / 2} \\
& \times\left\{\left(\Gamma^{c a e} C^{-1}\right)_{\alpha \beta}+\frac{2 R e\left[x_{14} x_{35}\right]}{x_{13} x_{45}}\left(\eta^{c e}\left(\gamma^{a} C^{-1}\right)_{\alpha \beta}-\eta^{a c}\left(\gamma^{e} C^{-1}\right)_{\alpha \beta}\right)\right\}, \\
I_{7}^{c}= & <: S_{\alpha}\left(x_{4}\right): S_{\beta}\left(x_{5}\right): \psi^{c}\left(x_{3}\right):>=2^{-1 / 2} x_{45}^{-3 / 4}\left(x_{34} x_{35}\right)^{-1 / 2}\left(\gamma^{c} C^{-1}\right)_{\alpha \beta} .
\end{aligned}
$$

One can show that the integrand is invariant under $\operatorname{SL}(2, R)$ transformation. Gauge fixing this symmetry by fixing the position of the open string vertex operators as

$$
x_{1}=0, \quad x_{2}=1, \quad x_{3} \rightarrow \infty, \quad d x_{1} d x_{2} d x_{3} \rightarrow x_{3}^{2} .
$$

one finds the following integral

$$
\int d^{2} z|1-z|^{a}|z|^{b}(z-\bar{z})^{c}(z+\bar{z})^{d},
$$

where $d=0,1,2$ and $a, b, c$ are given in terms of the Mandelstam variables,i.e.,

$$
s=-\left(k_{1}+k_{3}\right)^{2}, \quad t=-\left(k_{1}+k_{2}\right)^{2}, \quad u=-\left(k_{2}+k_{3}\right)^{2} .
$$


The region of integration is the upper half of the complex plane. For $d=0,1$ the result is given in [48] and for $d=2$ the result is given in [43]. Using those integrals one can write the amplitude (2.7) as

$$
\mathcal{A}^{\mathrm{AAAC}}=\mathcal{A}_{1}+\mathcal{A}_{2}+\mathcal{A}_{3}
$$

where

$$
\begin{aligned}
\mathcal{A}_{1} \sim & 2^{1 / 2} \xi_{1 a} \xi_{2 b} \xi_{3 d} k_{1 f} k_{2 e} \operatorname{Tr}\left(P_{-} H_{(n)} M_{p} \Gamma^{\text {dbeaf }}\right)(t+s+u) L_{1}, \\
\mathcal{A}_{2} \sim & 2^{-1 / 2} \operatorname{Tr}\left(P_{-} H_{(n)} M_{p} \Gamma^{d b a}\right)\left\{-t L_{2} \xi_{1 a} \xi_{2 b} \xi_{3 d}+\left[L _ { 2 } \left(-2 k_{1} \cdot \xi_{2} k_{2 b} \xi_{1 a} \xi_{3 d}-2 k_{2} \cdot \xi_{1} k_{1 a} \xi_{2 b} \xi_{3 d}\right.\right.\right. \\
& \left.\left.\left.-2 k_{2} \cdot \xi_{1} k_{2 a} \xi_{2 b} \xi_{3 d}+2 k_{1} \cdot \xi_{2} k_{1 a} \xi_{1 b} \xi_{3 d}+2 \xi_{1} \cdot \xi_{2} k_{1 a} k_{2 b} \xi_{3 d}\right)\right]-[3 \leftrightarrow 1]-[3 \leftrightarrow 2]\right\}, \\
\mathcal{A}_{3} \sim & 2^{-1 / 2} L_{1}\left\{\left[\operatorname { T r } ( P _ { - } H _ { ( n ) } M _ { p } \xi _ { 3 } \cdot \gamma ) \left(-2 t k_{3} \cdot \xi_{1} k_{3} \cdot \xi_{2}+2 u k_{3} \cdot \xi_{1} k_{1} \cdot \xi_{2}+2 s k_{2} \cdot \xi_{1} k_{3} \cdot \xi_{2}\right.\right.\right. \\
& \left.\left.+u s \xi_{1} \cdot \xi_{2}\right)\right]+[3 \leftrightarrow 2]+[3 \leftrightarrow 1]+\left(\left[\operatorname { T r } ( P _ { - } H _ { ( n ) } M _ { p } k _ { 2 } \cdot \gamma ) \left(-2 t k_{3} \cdot \xi_{1} \xi_{2} \cdot \xi_{3}\right.\right.\right. \\
& \left.\left.\left.\left.+2 u k_{1} \cdot \xi_{2} \xi_{1} \cdot \xi_{3}+2 s k_{2} \cdot \xi_{1} \xi_{3} \cdot \xi_{2}-2 u \xi_{1} \cdot \xi_{2} k_{1} \cdot \xi_{3}\right)\right]+[2 \leftrightarrow 1]\right)\right\} .
\end{aligned}
$$

where the functions $L_{1}, L_{2}$ are the following

$$
\begin{aligned}
& L_{1}=(2)^{-2(t+s+u)} \pi \frac{\Gamma\left(-u+\frac{1}{2}\right) \Gamma\left(-s+\frac{1}{2}\right) \Gamma\left(-t+\frac{1}{2}\right) \Gamma(-t-s-u)}{\Gamma(-u-t+1) \Gamma(-t-s+1) \Gamma(-s-u+1)}, \\
& L_{2}=(2)^{-2(t+s+u)} \pi \frac{\Gamma(-u+1) \Gamma(-s+1) \Gamma(-t) \Gamma\left(-t-s-u+\frac{1}{2}\right)}{\Gamma(-u-t+1) \Gamma(-t-s+1) \Gamma(-s-u+1)} .
\end{aligned}
$$

Since $H_{(n)}, M_{p}, \Gamma^{\text {dbeaf }}$ and $\Gamma^{\text {dea }}$ are totally antisymmetric combinations of the Gamma matrices, one can then understand that the amplitude is non zero for $p=n+4, p=n+2$ and $p=n$. From the poles of the gamma functions, one can easily see that the scattering amplitude has infinite massless poles and infinite number of massive poles. To compare this with the field theory, which has massless fields, one must expand the amplitude such that the massless poles of the field theory survive and all other poles vanish in the form of contact terms. In the next section we use the low energy limit expansion by sending all Mandelstam variables to zero.

\section{Momentum expansion}

We want to examine the limit of $\alpha^{\prime} \rightarrow 0$ of the above string amplitude. Using the momentum conservation along the world volume of brane, $k_{1}^{a}+k_{2}^{a}+k_{3}^{a}+p^{a}=0$, one finds the Mandelstam variables satisfy the following constraint

$$
s+t+u=-p_{a} p^{a} .
$$

It has been argued in [49], generally speaking that the momentum expansion of a S-matrix element should be around $\left(k_{i}+k_{j}\right)^{2} \rightarrow 0$ and/or $k_{i} \cdot k_{j} \rightarrow 0$. The case $\left(k_{i}+k_{j}\right)^{2} \rightarrow 0$ is when 
there is a massless pole in the $\left(k_{i}+k_{j}\right)^{2}$-channel. Notice that the amplitude $(2.1)$ must have only massless poles in the $\left(k_{1}+k_{2}\right)^{2},\left(k_{1}+k_{3}\right)^{2}$ and $\left(k_{2}+k_{3}\right)^{2}$-channels, so correct momentum expansion at the low energy limit for t-channel must be around

$$
\left(k_{1}+k_{2}\right)^{2} \rightarrow 0, \quad k_{1} \cdot k_{3} \rightarrow 0, \quad k_{2} \cdot k_{3} \rightarrow 0 .
$$

Also the correct momentum expansion for s, u-channels respectively are

$$
\begin{aligned}
\left(k_{1}+k_{3}\right)^{2} & \rightarrow 0, & k_{1} \cdot k_{2} & \rightarrow 0, \\
\left(k_{2}+k_{3}\right)^{2} & \rightarrow 0, & k_{1} \cdot k_{2} \cdot k_{3} \rightarrow 0, & k_{1} \cdot k_{3} \rightarrow 0,
\end{aligned}
$$

Using the on-shell relations $k_{1}^{2}=k_{2}^{2}=k_{3}^{2}=0$ one can rewrite them in terms of the Mandelstam variables as

$$
s \rightarrow 0, \quad t \rightarrow 0, \quad u \rightarrow 0 .
$$

Including the constraint (3.1), one should realize that $p_{a} p^{a} \rightarrow 0$ which is possible for D-branes. Therefore the S-matrix element can be evaluated for BPS branes.

Expansion of the functions $L_{1}, L_{2}$ around the above point is

$$
\begin{aligned}
L_{1}= & -\pi^{5 / 2}\left(\sum_{n=0}^{\infty} c_{n}(s+t+u)^{n}+\frac{\sum_{n, m=0}^{\infty} c_{n, m}\left[s^{n} u^{m}+s^{m} u^{n}\right]}{(t+s+u)}\right. \\
& \left.+\sum_{p, n, m=0}^{\infty} f_{p, n, m}(s+t+u)^{p}\left[(s+u)^{n}(s u)^{m}\right]\right), \\
L_{2}= & -\pi^{3 / 2}\left(\frac{1}{t} \sum_{n=-1}^{\infty} b_{n}(u+s)^{n+1}+\sum_{p, n, m=0}^{\infty} e_{p, n, m} t^{p}(s u)^{n}(s+u)^{m}\right) .
\end{aligned}
$$

where some of the coefficients $b_{n}, e_{p, n, m}, c_{n}, c_{n, m}$ and $f_{p, n, m}$ are

$$
\begin{aligned}
b_{-1} & =1, b_{0}=0, b_{1}=\frac{1}{6} \pi^{2}, b_{2}=2 \zeta(3), c_{0}=0, c_{1}=-\frac{\pi^{2}}{6}, \\
e_{2,0,0} & =e_{0,1,0}=2 \zeta(3), e_{1,0,0}=\frac{1}{6} \pi^{2}, e_{1,0,2}=\frac{19}{60} \pi^{4}, e_{1,0,1}=e_{0,0,2}=6 \zeta(3), \\
e_{0,0,1} & =\frac{1}{3} \pi^{2}, e_{3,0,0}=\frac{19}{360} \pi^{4}, e_{0,0,3}=e_{2,0,1}=\frac{19}{90} \pi^{4}, e_{1,1,0}=e_{0,1,1}=\frac{1}{30} \pi^{4}, \\
c_{2} & =-2 \xi(3), c_{1,1}=\frac{\pi^{2}}{6}, c_{0,0}=\frac{1}{2}, c_{3,1}=c_{1,3}=\frac{2}{15} \pi^{4}, c_{2,2}=\frac{1}{5} \pi^{4}, \\
c_{1,0} & =c_{0,1}=0, c_{3,0}=c_{0,3}=0, c_{2,0}=c_{0,2}=\frac{\pi^{2}}{6}, c_{1,2}=c_{2,1}=-4 \xi(3), \\
f_{0,1,0} & =\frac{\pi^{2}}{3}, f_{0,2,0}=-f_{1,1,0}=-6 \xi(3), f_{0,0,1}=-2 \xi(3), c_{4,0}=c_{0,4}=\frac{1}{15} \pi^{4} .
\end{aligned}
$$

Note that the coefficients $b_{n}$ are exactly the coefficients that appear in the momentum expansion of the S-matrix element of one RR, two gauge fields and one tachyon vertex operator [43]. Meanwhile $c_{n}, c_{n, m}, f_{p, n, m}$ are different from those coefficients which appeared in [43]. The function of $L_{1}$ has infinite massless poles in the $(t+s+u)$-channel and $L_{2}$ has infinite massless poles in the $t$-channel. These poles must be reproduced in field theory by appropriate couplings. Let us study each case separately. 


\section{Low energy field theory}

We are interested in the part of effective field theory of D-branes which includes only gauge fields. It should be possible to extract the necessary terms from the covariant Born-Infeld action constructed as the effective D-brane action in (1.5) . The Born-Infeld action is an action for all orders of $\alpha^{\prime}$ (see for more details [50-56]). The low energy nonabelian extension of the action was proposed to be the symmetrized trace of non-abelian generalization of Born-Infeld action (with flat background in the bulk) [57]. There, it was shown that defining non abelian Born-Infeld action with this trace produced the known results for the scattering of gauge fields up to fourth order in the field strengths [58]. However, there are some reasons which indicate the symmetrized trace prescription does not work for $F^{6}$ [59]. Then, it was proved that the symmetrized trace requires corrections at sixth order $[60,61]$. Using noncommutative field theory some efforts for the form of BI action were done [62-64].

The non-abelian field strength and covariant derivative of the gauge field are defined respectively as

$$
F^{a b}=\partial^{a} A^{b}-\partial^{b} A^{a}-i\left[A^{a}, A^{b}\right], \quad D_{a} F_{b c}=\partial_{a} F_{b c}-i\left[A_{a}, F_{b c}\right] .
$$

where $A_{a}=A_{a}^{\alpha} \Lambda_{\alpha}$ and $\Lambda_{\alpha}$ are the hermitian matrices. Our conventions for $\Lambda^{\alpha}$ are

$$
\sum_{\alpha} \Lambda_{i j}^{\alpha} \Lambda_{k l}^{\alpha}=\delta_{i k} \delta_{j l}, \quad \operatorname{Tr}\left(\Lambda^{\alpha} \Lambda^{\beta}\right)=\delta^{\alpha \beta} .
$$

Using the following expression, one can expand the square root in the non-abelian action (1.5) to produce various interacting terms [65] :

$$
\begin{aligned}
\sqrt{-\operatorname{det}\left(M_{0}+M\right)}= & \sqrt{-\operatorname{det}\left(M_{0}\right)}\left(1+\frac{1}{2} \operatorname{Tr}\left(M_{0}^{-1} M\right)-\frac{1}{4} \operatorname{Tr}\left(M_{0}^{-1} M M_{0}^{-1} M\right)\right. \\
& +\frac{1}{6} \operatorname{Tr}\left(M_{0}^{-1} M M_{0}^{-1} M M_{0}^{-1} M\right)-\frac{1}{8} \operatorname{Tr}\left(M_{0}^{-1} M M_{0}^{-1} M M_{0}^{-1} M M_{0}^{-1} M\right) \\
& +\frac{1}{8}\left(\operatorname{Tr}\left(M_{0}^{-1} M\right)\right)^{2}-\frac{1}{8}\left(\operatorname{Tr}\left(M_{0}^{-1} M\right)\right)^{3}+\frac{1}{32}\left(\operatorname{Tr}\left(M_{0}^{-1} M M_{0}^{-1} M\right)\right)^{2} \\
& +\cdots) .
\end{aligned}
$$

In (1.5), $M_{0}$ and $M$ are

$$
\begin{aligned}
M_{0} & =\eta_{a b}, \\
M & =2 \pi \alpha^{\prime} F_{a b} .
\end{aligned}
$$

The terms of the above expansion which have contribution to the S-matrix element (2.1) are in the following

$$
\begin{aligned}
\mathcal{L}= & -T_{p}\left(\pi \alpha^{\prime}\right) \operatorname{Tr}\left(-\left(\pi \alpha^{\prime}\right) F_{a b} F^{b a}\right) \\
& -T_{p}\left(2 \pi \alpha^{\prime}\right)^{4} S \operatorname{Tr}\left(-\frac{1}{8} F_{b d} F^{d f} F_{f h} F^{h b}+\frac{1}{32}\left(F_{a b} F^{b a}\right)^{2}\right) .
\end{aligned}
$$


Note that after averaging all possible permutations of the above terms in the second line (4.2), one must take overall trace over the group theory indices. The couplings in the second line have been confirmed [66]. We want to obtain the higher derivative couplings of the four gauge fields and then show that these terms reproduce infinite massless poles in the S-matrix.

\section{1 $p=n+4$ case}

This is the simplest case to consider. Only $\mathcal{A}_{1}$ in (2.10) is non-zero. One can calculate the trace of $\mathcal{A}_{1}$ as follows

$$
\operatorname{Tr}\left(P_{-} H_{(n)} M_{p} \Gamma^{d b e a f}\right)= \pm \frac{32}{2 n !} \epsilon^{d b e a f a_{0} \cdots a_{p-5}} H_{a_{0} \cdots a_{p-5}},
$$

We are going to compare string theory S-matrix elements with field theory including their coefficients, however we are not interested in fixing the overall sign of the amplitudes. Taking into account the above trace, the string amplitude becomes

$\mathcal{A}^{\mathrm{CAAA}}= \pm \frac{32}{(p-4) !} \mu_{p} \pi^{1 / 2} \operatorname{Tr}\left(\lambda_{1} \lambda_{2} \lambda_{3}\right) \xi_{1 a} \xi_{2 b} \xi_{3 d} k_{1 f} k_{2 e} \epsilon^{d b e a f a_{0} \cdots a_{p-5}} H_{a_{0} \cdots a_{p-5}}(s+t+u) L_{1}$,

where we normalized the amplitude by $\left(\mu_{p} 2^{1 / 2} \pi^{1 / 2}\right)$. The above amplitude is antisymmetric upon interchanging the gauge fields. So the whole amplitude is zero for an abelian gauge group. The amplitude also satisfies the Ward identity, i.e., it vanishes under replacing each of $\xi^{i} \rightarrow k^{i}$. Since $(t+s+u) L_{1}$ has no tachyon/massless pole, then the amplitude has only contact terms. The leading contact term is reproduced by the following coupling

$$
\frac{1}{3 !} \mu_{p}\left(2 \pi \alpha^{\prime}\right)^{3} \operatorname{Tr}\left(C_{p-5} \wedge F \wedge F \wedge F\right) .
$$

The non-leading order terms should correspond to the higher derivative extension of the coupling.

\section{$4.2 p=n$ case}

The next simple case is $p=n$. Only $\mathcal{A}_{3}$ in (2.10) is non-zero for this case. The calculation of the trace in this part of the amplitude is

$$
\operatorname{Tr}\left(H_{(n)} M_{p} \gamma^{a}\right)= \pm \frac{32}{n !} \epsilon^{a_{0} \cdots a_{p-1} a} H_{a_{0} \cdots a_{p-1}},
$$

Substituting this trace in $\mathcal{A}_{3}$, one finds

$$
\begin{aligned}
\mathcal{A}^{\mathrm{CAAA}}= & \pm \frac{32}{2 p !} \mu_{p} \pi^{1 / 2} L_{1} \operatorname{Tr}\left(\lambda_{1} \lambda_{2} \lambda_{3}\right) \epsilon^{a_{0} \cdots a_{p-1} a} H_{a_{0} \cdots a_{p-1}}\left\{\left[\xi _ { 3 a } \left(-2 t k_{3} \cdot \xi_{1} k_{3} \cdot \xi_{2}+2 u k_{3} \cdot \xi_{1}\right.\right.\right. \\
& \left.\left.\times k_{1} \cdot \xi_{2}+2 s k_{2} \cdot \xi_{1} k_{3} \cdot \xi_{2}+u s \xi_{1} \cdot \xi_{2}\right)\right]+[3 \leftrightarrow 2]+[3 \leftrightarrow 1]+\left(\left[k _ { 2 a } \left(-2 t k_{3} \cdot \xi_{1} \xi_{2} \cdot \xi_{3}\right.\right.\right. \\
& \left.\left.\left.\left.+2 u k_{1} \cdot \xi_{2} \xi_{1} \cdot \xi_{3}+2 s k_{2} \cdot \xi_{1} \xi_{3} \cdot \xi_{2}-2 u \xi_{1} \cdot \xi_{2} k_{1} \cdot \xi_{3}\right)\right]+[2 \leftrightarrow 1]\right)\right\} .
\end{aligned}
$$




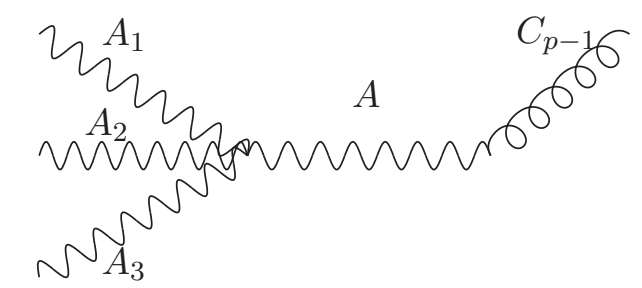

Figure 1. The Feynman diagram corresponding to the amplitudes (4.5).

A check of our calculations is that the above amplitude satisfies the Ward identity associated with the gauge invariance of the open string states. The amplitude is symmetric under interchanging $1 \leftrightarrow 2$. So the amplitude is non-zero even for the abelian case. All terms in (4.5) have infinite massless poles in the $(s+t+u)$-channel and infinite contact terms. In the next section, firstly we want to reproduce the first massless pole using the symmetric trace prescription of BI action. Then we find higher derivative couplings of four gauge fields in order to show that massless poles can be produced to all orders of $\alpha^{\prime}$ by WZ coupling $C_{p} \wedge F$ and by the higher derivative couplings of four gauge fields.

\subsection{First massless pole for $p=n$ case}

The terms in the second line of (4.2) give four gauge field couplings. In order to reproduce the first massless pole from the couplings (4.2) we should consider figure 1 as the Feynman diagram for $p=n$ case. Since the propagator is abelian, we must calculate three possible permutations to obtain the desired 123 ordering. Writing symmetric traces in terms of ordinary traces (apart from overall factor), one can write the two last terms in (4.2) as $\left(L_{5}^{0,0}+L_{6}^{0,0}+L_{7}^{0,0}\right)$ which are the following :

$$
\begin{aligned}
& L_{5}^{0,0}=-\frac{1}{4 \pi^{2}} \operatorname{Tr}\left(a_{0,0}\left(F_{b d} F^{d f} F_{f h} F^{h b}\right)+b_{0,0}\left(F_{b d} F_{f h} F^{d f} F^{h b}\right)\right), \\
& L_{6}^{0,0}=-\frac{1}{4 \pi^{2}} \operatorname{Tr}\left(a_{0,0}\left(F_{b d} F^{d f} F^{h b} F_{f h}\right)+b_{0,0}\left(F_{b d} F^{h b} F^{d f} F_{f h}\right)\right), \\
& L_{7}^{0,0}=\frac{1}{8 \pi^{2}} \operatorname{Tr}\left(a_{0,0}\left(F_{a b} F^{a b} F_{c d} F^{c d}\right)+b_{0,0}\left(F_{a b} F^{c d} F^{a b} F_{c d}\right)\right) .
\end{aligned}
$$

where $a_{0,0}=\frac{-\pi^{2}}{6}, b_{0,0}=\frac{-\pi^{2}}{12}$.

The massless poles of the amplitude (4.5) are given by the following amplitude

$$
\mathcal{A}=V_{\alpha}^{a}\left(C_{p-1}, A\right) G_{\alpha \beta}^{a b}(A) V_{\beta}^{b}\left(A, A_{1}, A_{2}, A_{3}\right)
$$


where the gauge field propagator and the vertex $V_{\alpha}^{a}\left(C_{p-1}, A\right)$ are given as

$$
\begin{aligned}
G_{\alpha \beta}^{a b}(A) & =\frac{i \delta_{\alpha \beta} \delta^{a b}}{\left(2 \pi \alpha^{\prime}\right)^{2} T_{p}(s+t+u)}, \\
V_{\alpha}^{a}\left(C_{p-1}, A\right) & =i\left(2 \pi \alpha^{\prime}\right) \mu_{p} \frac{1}{p !} \epsilon^{a_{0} \cdots a_{p-1} a} H_{a_{0} \cdots a_{p-1}} \operatorname{Tr}\left(\Lambda_{\alpha}\right) .
\end{aligned}
$$

where $V_{\alpha}^{a}\left(C_{p-1}, A\right)$ has been found in [42]. In the above vertex $\operatorname{Tr}\left(\Lambda_{\alpha}\right)$ is non-zero for the abelian matrix $\Lambda_{\alpha}$. The vertex $V_{\beta}^{b}\left(A, A_{1}, A_{2}, A_{3}\right)$ can be obtained from the four gauge field couplings of (4.6) as follows

$$
\begin{aligned}
I_{8}= & V_{\beta}^{b}\left(A, A_{1}, A_{2}, A_{3}\right)=\left(2 \pi \alpha^{\prime}\right)^{4} T_{p} \frac{1}{4} \operatorname{Tr}\left(\lambda_{1} \lambda_{2} \lambda_{3} \Lambda_{\beta}\right)\left\{\xi _ { 3 } ^ { b } \left[2 t k_{3} \cdot \xi_{1} k_{3} \cdot \xi_{2}-2 u k_{3} \cdot \xi_{1} k_{1} \cdot \xi_{2}\right.\right. \\
& \left.-2 s k_{2} \cdot \xi_{1} k_{3} \cdot \xi_{2}-u s \xi_{1} \cdot \xi_{2}\right]+[3 \leftrightarrow 2]+[3 \leftrightarrow 1]+\left(k _ { 2 } ^ { b } \left[+2 t k_{3} \cdot \xi_{1} \xi_{2} \cdot \xi_{3}-2 u k_{1} \cdot \xi_{2} \xi_{1} \cdot \xi_{3}\right.\right. \\
& \left.\left.\left.+2 u k_{1} \cdot \xi_{3} \xi_{1} \cdot \xi_{2}-2 s \xi_{2} \cdot \xi_{3} k_{2} \cdot \xi_{1}\right]+[2 \leftrightarrow 1]\right)\right\}
\end{aligned}
$$

where $k_{1}, k_{2}, k_{3}$ are the momenta of on-shell gauge fields. Replacing the above vertex in (4.7) we find exactly the first massless pole of the equation of (4.5). In order to obtain all infinite massless poles of the amplitude for the $p=n$ case we should find higher derivative couplings of four gauge fields.

\section{$5 \quad$ Four gauge field couplings}

The S-matrix element of all four point massless vertex operators in superstring theory was calculated in standard books $[67,68]$. For two important reasons one can find the higher derivative couplings of four gauge fields from higher derivative couplings of four scalar fields [38] by using T-duality transformation.

The first reason is that Mandelstam Variables for both four gauge fields amplitude and four massless scalar fields amplitude satisfy the constraint of $s+t+u=0$. Also the massless poles of the Feynman amplitude resulting from the non abelian kinetic term of the scalars and gauge fields are reproduced at the low energy limit by sending $s, t, u \rightarrow 0$. The second reason is that, external states in both of them satisfy the on-shell condition $k_{i}^{2}=0$ and physical state condition $k \cdot \xi=0$ (in fact, the behavior of massless transverse scalars is similar to world volume gauge fields). Also both of them transform in the adjoint representation of $\mathrm{U}(N)$ group.

One may expect that the higher derivative couplings of four gauge fields should be similar to the higher derivative couplings of four scalar fields. The only difference is related to their polarization. Gauge fields' polarization only has components in the world volume direction while scalar fields' polarization has transverse components on the D-brane i.e., the physical state condition for gauge field is $k_{1} \cdot \xi_{1}=k_{2} \cdot \xi_{2}=\cdots=k_{n} \cdot \xi_{n}=0$ while for scalar fields it satisfies $k_{1} \cdot \xi_{1}=k_{1} \cdot \xi_{2}=\cdots=k_{i} \cdot \xi_{j}=0$ where $i, j=1,2, \ldots n$.

On the other hand the S-matrix element of the scalar field vertex operators can be read from the S-matrix element of gauge field vertex operators by restricting the polarization 
of the gauge fields to transverse directions and the momentum of the gauge fields to the world-volume directions. To find four gauge field couplings to all orders of $\alpha^{\prime}$, we follow the steps mentioned in [38]. The massless poles in (4.5) are reproduced by the non-abelian kinetic terms of the gauge field and the contact terms with coefficients $a_{0,0}$ and $b_{0,0}$ are also reproduced (apart from an over all factor) by the following terms

$$
-T_{p} S \operatorname{Tr}\left(-\frac{1}{8} F_{b d} F^{d f} F_{f h} F^{h b}+\frac{1}{32}\left(F_{a b} F^{b a}\right)^{2}\right) .
$$

Meanwhile for the scalar field they are reproduced by

$$
-T_{p} \operatorname{STr}\left(-\frac{1}{4} D_{a} \phi^{i} D_{b} \phi_{i} D^{b} \phi^{j} D^{a} \phi_{j}+\frac{1}{8}\left(D_{a} \phi^{i} D^{a} \phi_{i}\right)^{2}\right) .
$$

Note that the differences between (5.1) and (5.2) are the coefficients and indices. Therefore using T-duality transformation not only should one substitute the covariant derivative of the scalar field $D \phi$ into the field strength of the gauge field $F$, but also replace transverse indices with world volume indices to find higher derivative couplings of four gauge fields from higher derivative couplings of four scalar fields (the equation (35) of [38]). Performing symmetric traces in terms of ordinary traces one can write (5.1) as (4.6). Now one can extend it to the higher derivative terms as

$$
\left(2 \pi \alpha^{\prime}\right)^{4} \frac{1}{8 \pi^{2}} T_{p}\left(\alpha^{\prime}\right)^{n+m} \sum_{m, n=0}^{\infty}\left(\mathcal{L}_{5}^{n m}+\mathcal{L}_{6}^{n m}+\mathcal{L}_{7}^{n m}\right),
$$

with

$$
\begin{aligned}
& \mathcal{L}_{5}^{n m}=-\operatorname{Tr}\left(a_{n, m} \mathcal{D}_{n m}\left[F_{b d} F^{d f} F_{f h} F^{h b}\right]+b_{n, m} \mathcal{D}_{n m}^{\prime}\left[F_{b d} F_{f h} F^{d f} F^{h b}\right]+h . c .\right), \\
& \mathcal{L}_{6}^{n m}=-\operatorname{Tr}\left(a_{n, m} \mathcal{D}_{n m}\left[F_{b d} F^{d f} F^{h b} F_{f h}\right]+b_{n, m} \mathcal{D}_{n m}^{\prime}\left[F_{b d} F^{h b} F^{d f} F_{f h}\right]+h . c .\right), \\
& \mathcal{L}_{7}^{n m}=\frac{1}{2} \operatorname{Tr}\left(a_{n, m} \mathcal{D}_{n m}\left[F_{a b} F^{a b} F_{c d} F^{c d}\right]+b_{n, m} \mathcal{D}_{n m}^{\prime}\left[F_{a b} F^{c d} F^{a b} F_{c d}\right]+h . c\right),
\end{aligned}
$$

where the higher derivative operators $D_{n m}$ and $D_{n m}^{\prime}$ are defined [38] as

$$
\begin{aligned}
& \mathcal{D}_{n m}(E F G H) \equiv D_{b_{1}} \cdots D_{b_{m}} D_{a_{1}} \cdots D_{a_{n}} E F D^{a_{1}} \cdots D^{a_{n}} G D^{b_{1}} \cdots D^{b_{m}} H \\
& \mathcal{D}_{n m}^{\prime}(E F G H) \equiv D_{b_{1}} \cdots D_{b_{m}} D_{a_{1}} \cdots D_{a_{n}} E D^{a_{1}} \cdots D^{a_{n}} F G D^{b_{1}} \cdots D^{b_{m}} H .
\end{aligned}
$$

Of course the above couplings are exact up to total derivative terms and terms like $\partial_{a} \partial^{a} F F F F$ which are zero on-shell. Also these terms have no effect on the massless poles of S-matrix elements because by canceling $k^{2}$ with the massless propagator one finds a contact term. These are the higher derivative extensions of four gauge field couplings of the action (1.5).

\subsection{Infinite massless poles for $p=n$ case}

Here we would like to check that the infinite four gauge field couplings (5.3) produce infinite massless poles of the string theory S-matrix element (4.5) which are in the $(s+t+u)$-channel. 
In fact they can be reproduced by WZ coupling $C_{p} \wedge F$ and by the higher derivative four gauge field couplings that have been found in (5.3). For this aim, consider the amplitude of the decay of one R-R field to three gauge fields in the world-volume theory of the BPS branes which is given by the Feynman amplitude (4.7), where the gauge field propagator and the vertex $V_{\alpha}^{a}\left(C_{p-1}, A\right)$ are in (4.8). According to the fact that the off-shell gauge field must be abelian, one finds the higher derivative vertex $V_{\beta}^{b}\left(A, A_{1}, A_{2}, A_{3}\right)$ from the higher derivative couplings in (5.3) to be

$$
\begin{aligned}
& \frac{I_{8}}{2 \pi^{2}}\left(\alpha^{\prime}\right)^{n+m}\left(a_{n, m}+b_{n, m}\right)\left(\left(k_{3} \cdot k_{1}\right)^{n}\left(k_{1} \cdot k\right)^{m}+\left(k_{1} \cdot k\right)^{m}\left(k_{2} \cdot k\right)^{n}+\left(k_{1} \cdot k\right)^{n}\left(k_{1} \cdot k_{3}\right)^{m}\right. \\
& \quad+\left(k_{1} \cdot k_{3}\right)^{m}\left(k_{3} \cdot k_{2}\right)^{n}+\left(k_{3} \cdot k_{1}\right)^{n}\left(k_{2} \cdot k_{3}\right)^{m}+\left(k_{3} \cdot k_{2}\right)^{m}\left(k_{2} \cdot k\right)^{n}+\left(k_{1} \cdot k\right)^{n}\left(k_{2} \cdot k\right)^{m} \\
& \left.\quad+\left(k_{3} \cdot k_{2}\right)^{n}\left(k_{2} \cdot k\right)^{m}\right)
\end{aligned}
$$

where $I_{8}$ is in (4.9) and $k$ is the momentum of the off-shell gauge field. Note that we must consider all 12 possible cyclic permutations to obtain the desired 123 ordering of the amplitude. Some of the coefficients $a_{n, m}$ and $b_{n, m}$ are [38]

$$
\begin{aligned}
& a_{0,0}=-\frac{\pi^{2}}{6}, b_{0,0}=-\frac{\pi^{2}}{12}, a_{1,0}=2 \zeta(3), a_{0,1}=0, b_{0,1}=-\zeta(3), a_{1,1}=a_{0,2}=-7 \pi^{4} / 90, \\
& a_{2,2}=\left(-83 \pi^{6}-7560 \zeta(3)^{2}\right) / 945, b_{2,2}=-\left(23 \pi^{6}-15120 \zeta(3)^{2}\right) / 1890, a_{1,3}=-62 \pi^{6} / 945, \\
& a_{2,0}=-4 \pi^{4} / 90, b_{1,1}=-\pi^{4} / 180, b_{0,2}=-\pi^{4} / 45, a_{0,4}=-31 \pi^{6} / 945, a_{4,0}=-16 \pi^{6} / 945, \\
& a_{1,2}=a_{2,1}=8 \zeta(5)+4 \pi^{2} \zeta(3) / 3, a_{0,3}=0, a_{3,0}=8 \zeta(5), b_{1,3}=-\left(12 \pi^{6}-7560 \zeta(3)^{2}\right) / 1890, \\
& a_{3,1}=\left(-52 \pi^{6}-7560 \zeta(3)^{2}\right) / 945, b_{0,3}=-4 \zeta(5), b_{1,2}=-8 \zeta(5)+2 \pi^{2} \zeta(3) / 3, \\
& b_{0,4}=-16 \pi^{6} / 1890 .
\end{aligned}
$$

where $b_{n, m}$ is symmetric.

Now one can write $k_{1} \cdot k=k_{2} \cdot k_{3}-\left(k^{2}\right) / 2$ and $k_{2} \cdot k=k_{1} \cdot k_{3}-\left(k^{2}\right) / 2$. The terms $k^{2}$ in the vertex (5.4) will be canceled with the $k^{2}$ in the denominator of the gauge field propagator producing a bunch of contact terms of one $\mathrm{RR}$ and three gauge fields which we are not interested in considering. Neglecting them, one finds the following infinite massless poles

$$
\begin{aligned}
& -32 \pi \mu_{p} \frac{\epsilon^{a_{0} \cdots a_{p-1} a} H_{a_{0} \cdots a_{p-1}}}{p !(s+t+u)} \operatorname{Tr}\left(\lambda_{1} \lambda_{2} \lambda_{3}\right) \sum_{n, m=0}^{\infty}\left(( a _ { n , m } + b _ { n , m } ) [ s ^ { m } u ^ { n } + s ^ { n } u ^ { m } ] \left\{\left[\xi _ { 3 a } \left(-2 t k_{3} \cdot \xi_{1}\right.\right.\right.\right. \\
& \left.\left.\times k_{3} \cdot \xi_{2}+2 u k_{3} \cdot \xi_{1} k_{1} \cdot \xi_{2}+2 s k_{2} \cdot \xi_{1} k_{3} \cdot \xi_{2}+u s \xi_{1} \cdot \xi_{2}\right)\right]+[3 \leftrightarrow 2]+[3 \leftrightarrow 1]+\left(\left[k _ { 2 a } \left(-2 t k_{3} \cdot \xi_{1}\right.\right.\right. \\
& \left.\left.\left.\left.\times \xi_{2} \cdot \xi_{3}+2 u k_{1} \cdot \xi_{2} \xi_{1} \cdot \xi_{3}+2 s k_{2} \cdot \xi_{1} \xi_{3} \cdot \xi_{2}-2 u \xi_{1} \cdot \xi_{2} k_{1} \cdot \xi_{3}\right)\right]+[2 \leftrightarrow 1]\right)\right\} .
\end{aligned}
$$

As a check of our calculations let us compare the above amplitude with the massless poles in (4.5) for some values of $n, m$. For $n=m=0$, the amplitude (5.6) has the following numerical factor

$$
-8\left(a_{0,0}+b_{0,0}\right)=-8\left(\frac{-\pi^{2}}{6}+\frac{-\pi^{2}}{12}\right)=2 \pi^{2}
$$


A similar term in (4.5) has the numerical factor $\left(4 \pi^{2} c_{0,0}\right)$ which is equal to the above number. At the order of $\alpha^{\prime}$, the amplitude (5.6) has the following numerical factor

$$
-4\left(a_{1,0}+a_{0,1}+b_{1,0}+b_{0,1}\right)(s+u)=0
$$

A similar term in (4.5) is proportional to $2 \pi^{2}\left(c_{1,0}+c_{0,1}\right)(s+u)$ which is zero. At the order of $\left(\alpha^{\prime}\right)^{2}$, the amplitude (5.6) has the following factor

$$
-8\left(a_{1,1}+b_{1,1}\right) s u-4\left(a_{0,2}+a_{2,0}+b_{0,2}+b_{2,0}\right)\left[s^{2}+u^{2}\right]=\frac{\pi^{4}}{3}(2 s u)+\frac{2 \pi^{4}}{3}\left(s^{2}+u^{2}\right)
$$

A similar term in (4.5) has the numerical factor $2 \pi^{2}\left[c_{1,1}(2 s u)+\left(c_{2,0}+c_{0,2}\right)\left(s^{2}+u^{2}\right)\right]$ which is equal to the above factor using the coefficients in (3.4). At the order of $\alpha^{\prime 3}$, this amplitude has the following factor

$$
\begin{aligned}
& -4\left(a_{3,0}+a_{0,3}+b_{0,3}+b_{3,0}\right)\left[s^{3}+u^{3}\right]-4\left(a_{1,2}+a_{2,1}+b_{1,2}+b_{2,1}\right)[s u(s+u)] \\
& \quad=-16 \pi^{2} \xi(3) s u(s+u)
\end{aligned}
$$

which is equal to the corresponding term in (4.5), i.e., $2 \pi^{2}\left[\left(c_{0,3}+c_{3,0}\right)\left[s^{3}+u^{3}\right]+\left(c_{2,1}+\right.\right.$ $\left.\left.c_{1,2}\right) s u(s+u)\right]$. At the order of $\left(\alpha^{\prime}\right)^{4}$, the amplitude (5.6) has the following factor

$$
\begin{gathered}
-4\left(a_{4,0}+a_{0,4}+b_{0,4}+b_{4,0}\right)\left(s^{4}+u^{4}\right)-4\left(a_{3,1}+a_{1,3}+b_{3,1}+b_{1,3}\right)\left[s u\left(s^{2}+u^{2}\right)\right] \\
-8\left(a_{2,2}+b_{2,2}\right) s^{2} u^{2}=\frac{4 \pi^{6}}{15}\left(s^{4}+u^{4}+2\left(s^{3} u+u^{3} s\right)+3 s^{2} u^{2}\right)
\end{gathered}
$$

A similar term in (4.5) has the numerical factor $2 \pi^{2}\left[\left(c_{4,0}+c_{0,4}\right)\left(s^{4}+u^{4}\right)+\left(c_{1,3}+c_{3,1}\right)\left(s^{3} u+\right.\right.$ $\left.\left.u^{3} s\right)+2 c_{2,2} s^{2} u^{2}\right]$ which is equal to the above factor using the coefficients in (3.4). We have reproduced the known results for terms at $\mathcal{O}\left(\alpha^{\prime 4}\right){ }^{2}$ A similar comparison can be done for all orders of $\alpha^{\prime}$. Hence, the field theory amplitude (5.6) reproduces exactly the infinite massless poles of the string theory amplitude (4.5). This shows that in addition to higher derivative couplings of four gauge fields being exact up to zero on-shell, they are also consistent with the momentum expansion of the amplitude $C A A A$.

\subsection{Infinite massless poles for $p=n+2$ case}

The last case is $p=n+2$. Only $\mathcal{A}_{2}$ in $(2.10)$ is non-zero for this case. The trace is calculated as follows

$$
\operatorname{Tr}\left(H_{(n)} M_{p} \Gamma^{d b a}\right)= \pm \frac{32}{n !} \epsilon^{a_{0} \cdots a_{p-3} d b a} H_{a_{0} \cdots a_{p-3}},
$$

\footnotetext{
${ }^{2}$ Our results up to $\mathcal{O}\left(\alpha^{\prime 4}\right)$ are consistent with those terms which found in [69-71] up to on-shell ambiguity and total derivative terms.
} 


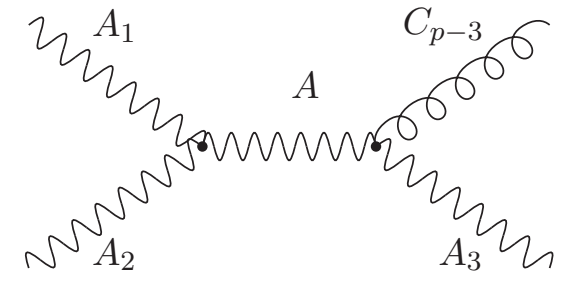

(a)

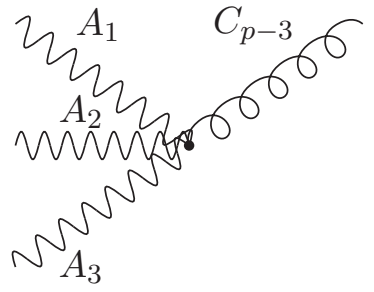

(b)

Figure 2. The Feynman diagrams (a) and (b) corresponding to the massless pole of the amplitude (5.7) and the couplings (5.9).

Replacing (3.3) and the above trace in the second part of the amplitude (2.10), one finds the electric part of the amplitude for $p=n+2$ case is given by

$$
\begin{aligned}
A^{\mathrm{CAAA}}= & \mp \frac{32}{2(p-2) !} \mu_{p} \pi^{2} \operatorname{Tr}\left(\lambda_{1} \lambda_{2} \lambda_{3}\right) \epsilon^{a_{0} \cdots a_{p-3} d e a} H_{a_{0} \cdots a_{p-3}}\left\{-\sum_{n=-1}^{\infty} b_{n}(u+s)^{n+1} \xi_{3 d} \xi_{2 e} \xi_{1 a}\right. \\
& +\left(\left[\sum _ { n = - 1 } ^ { \infty } \frac { 1 } { t } b _ { n } ( u + s ) ^ { n + 1 } \left(-2 k_{1} \cdot \xi_{2} k_{2 e} \xi_{1 a} \xi_{3 d}-2 k_{2} \cdot \xi_{1} k_{1 a} \xi_{2 e} \xi_{3 d}-2 k_{2} \cdot \xi_{1} k_{2 a} \xi_{2 e} \xi_{3 d}\right.\right.\right. \\
& \left.\left.\left.\left.+2 k_{1} \cdot \xi_{2} k_{1 a} \xi_{1 e} \xi_{3 d}+2 \xi_{1} \cdot \xi_{2} k_{1 a} k_{2 e} \xi_{3 d}\right)\right]-[3 \leftrightarrow 1]-[3 \leftrightarrow 2]\right)\right\}
\end{aligned}
$$

The amplitude is antisymmetric under the interchange of the gauge fields, so the whole amplitude is zero for the abelian gauge group. The amplitude satisfies the Ward identity for all three gauge fields. Note that only the first term is related to the infinite contact terms while the other terms are indeed infinite massless poles. We are going to analyze all orders of the massless poles in this section and the leading order and next to leading order of the contact terms in the following section.

The amplitudes in s,u and t-channels are very similar, so we analyze the amplitude with whole details only in t-channel. Infinite massless poles in the t-channel should be reproduced in field theory according to the Feynman diagram of figure 2(a).

Therefore the infinite massless poles are given by the Feynman amplitude

$$
\mathcal{A}=V_{\alpha}^{a}\left(C_{p-3}, A_{3}, A\right) G_{\alpha \beta}^{a b}(A) V_{\beta}^{b}\left(A, A_{1}, A_{2}\right),
$$

where the vertices and propagator are

$$
\begin{aligned}
V_{\alpha}^{a}\left(C_{p-3}, A_{3}, A\right) & =\frac{\mu_{p}\left(2 \pi \alpha^{\prime}\right)^{2}}{(p-2) !} \epsilon^{a \cdots \cdots a_{p-1} a} H_{a_{0} \cdots a_{p-3}} \xi_{3 a_{p-2}} k_{a_{p-1}} \operatorname{Tr}\left(\lambda_{3} \Lambda_{\alpha}\right) \sum_{n=-1}^{\infty} b_{n}\left(\alpha^{\prime} k_{3} \cdot k\right)^{n+1}, \\
V_{\beta}^{b}\left(A, A_{1}, A_{2}\right) & =-i T_{p}\left(2 \pi \alpha^{\prime}\right)^{2} \operatorname{Tr}\left(\lambda_{1} \lambda_{2} \Lambda_{\beta}\right)\left[\xi_{1}^{b}\left(k_{1}-k\right) \cdot \xi_{2}+\xi_{2}^{b}\left(k-k_{2}\right) \cdot \xi_{1}+\xi_{1} \cdot \xi_{2}\left(k_{2}-k_{1}\right)^{b}\right], \\
G_{\alpha \beta}^{a b}(A) & =\frac{i \delta_{\alpha \beta} \delta^{a b}}{\left(2 \pi \alpha^{\prime}\right)^{2} T_{p}(t)},
\end{aligned}
$$


where the propagator is derived from the standard gauge kinetic term arising in the expansion of the Born-Infeld action. Note that the vertex $V_{\beta}^{b}\left(A, A_{1}, A_{2}\right)$ is found from the standard non abelian kinetic term of the gauge field, and also the vertex $V_{\alpha}^{a}\left(C_{p-3}, A_{3}, A\right)$ is found from the higher derivative extension of the WZ coupling $C_{p-3} \wedge F \wedge F$ [49]. In the above formula $k$ is the momentum of the off-shell gauge field. The important point is that the vertex $V_{\beta}^{b}\left(A, A_{1}, A_{2}\right)$ has no higher derivative correction as it arises from the kinetic term of the gauge field. This vertex has already been found in [43]. Substituting them to the amplitude (5.8) becomes

$$
\begin{aligned}
\mathcal{A}= & \mu_{p}\left(2 \pi \alpha^{\prime}\right)^{2} \frac{1}{(p-2) ! t} \operatorname{Tr}\left(\lambda_{1} \lambda_{2} \lambda_{3}\right) \epsilon^{a_{0} \cdots a_{p-1} a} H_{a_{0} \cdots a_{p-3}} \xi_{3 a_{p-2}} \sum_{n=-1}^{\infty} b_{n}\left(\frac{\alpha^{\prime}}{2}\right)^{n+1}(s+u)^{n+1} \\
& \times\left[-2 k_{1} \cdot \xi_{2} k_{1 a_{p-1}} \xi_{1 a}-2 k_{1} \cdot \xi_{2} k_{2 a_{p-1}} \xi_{1 a}+2 k_{2} \cdot \xi_{1} k_{1 a_{p-1}} \xi_{2 a}+2 k_{2} \cdot \xi_{1} k_{2 a_{p-1}} \xi_{2 a}\right. \\
& \left.-2 \xi_{1} \cdot \xi_{2} k_{2 a} k_{1 a_{p-1}}\right] .
\end{aligned}
$$

which describes exactly the same infinite massless poles of the string theory amplitude (5.7) in t-channel.

\subsection{Contact terms}

After finding all infinite massless poles, we now extract the low energy contact terms of the string amplitude for $p=n+2$ case. Substituting (3.3) into (5.7), one finds the following contact terms at leading order and next to the leading order

$$
\begin{aligned}
A^{\mathrm{CAAA}}= & \mp \frac{32 \mu_{p} \pi^{2}}{2(p-2) !} \operatorname{Tr}\left(\lambda_{1} \lambda_{2} \lambda_{3}\right) \epsilon^{a_{0} \cdots a_{p-3} d e a} H_{a_{0} \cdots a_{p-3}}\left\{\xi_{3 d} \xi_{2 e} \xi_{1 a}+\frac{\pi^{2}}{6} \xi_{3 d} \xi_{2 e} \xi_{1 a}\left[(s+u)^{2}\right.\right. \\
& +t(t+2 s+2 u)]-\left(\left[\frac { \pi ^ { 2 } } { 6 } ( t + 2 s + 2 u ) \left(-2 k_{1} \cdot \xi_{2} k_{2 e} \xi_{1 a} \xi_{3 d}-2 k_{2} \cdot \xi_{1} k_{1 a} \xi_{2 e} \xi_{3 d}\right.\right.\right. \\
& \left.\left.-2 k_{2} \cdot \xi_{1} k_{2 a} \xi_{2 e} \xi_{3 d}+2 k_{1} \cdot \xi_{2} k_{1 a} \xi_{1 e} \xi_{3 d}+2 \xi_{1} \cdot \xi_{2} k_{1 a} k_{2 e} \xi_{3 d}\right)\right]-[3 \leftrightarrow 1] \\
& -[3 \leftrightarrow 2])\} .
\end{aligned}
$$

The first term is reproduced by $C A A A$ coupling of the following gauge invariant coupling

$$
\frac{\mu_{p}}{2 !}\left(2 \pi \alpha^{\prime}\right)^{2} \operatorname{Tr}\left(C_{p-3} \wedge F \wedge F\right),
$$

which is given exactly by the WZ terms in (1.3) after expanding the exponential. The other terms in (5.9) should be related to the higher derivative extension of the above coupling. However, there are various higher derivative gauge invariant couplings which make a contribution to the contact terms of the S-matrix element of $C A A A$. Comparing them with the string theory contact terms in (5.9), one can not fix all their coefficients uniquely. One particular set of higher derivative gauge invariant couplings that reproduces 
the contact terms in (5.9) is as follows:

$$
\begin{aligned}
& \frac{\mu_{p}\left(2 \pi \alpha^{\prime}\right)^{2} \pi^{2}}{6(p-3) !} \epsilon^{a_{0} \cdots a_{p-4} a b c d} C_{a_{0} \cdots a_{p-4}}\left[-\frac{1}{2} D^{\alpha} D^{\beta} F_{a b} D_{\alpha} D_{\beta} F_{c d}-D_{\alpha} F_{\beta b} D^{\alpha} D_{a} D^{\beta} F_{c d}\right. \\
& +3 D_{b} D^{\alpha} D^{\beta} F_{a \alpha} D_{\beta} F_{c d}-\frac{3}{2} D_{a} D^{\alpha} D^{\beta} D_{\beta} F_{b \alpha} F_{c d}-10 D^{\alpha} D^{\beta} D_{d} F_{a \alpha} D_{c} F_{b \beta} \\
& \left.-4 D_{c} F_{a \alpha} D^{\alpha} D^{\beta} D_{d} F_{b \beta}-\frac{3}{4} D^{\alpha} D_{\beta} D_{\alpha} D^{\beta} F_{a b} F_{c d}-6 D^{\beta} F_{a \alpha} D^{\alpha} D_{c} D_{d} F_{b \beta}\right] .
\end{aligned}
$$

where $D_{a} F=\partial_{a} F-i\left[A_{a}, F\right]$. Among the couplings in (5.11), only the first coupling has non-zero on-shell $C A A$ coupling. This coupling is obtained from the S-matrix element of one RR and two gauge field vertex operators. This coupling has also been used in the previous section to verify that the infinite massless poles (5.7) are reproduced by the higher derivative couplings in field theory. All couplings in (5.11) are at $\left(\alpha^{\prime}\right)^{4}$ order. The next order terms should be at $\left(\alpha^{\prime}\right)^{5}$ order, and so on. Hence, the leading order terms of the momentum expansion of the S-matrix element (2.10) correspond to the Feynman amplitudes resulting from $\mathrm{BI}$ and $\mathrm{WZ}$ actions and the higher order terms correspond to the higher derivative corrections to the WZ couplings. Note that the above higher derivative WZ couplings are valid when $p_{a} p^{a} \rightarrow 0$. Hence, they can not be compared with 'constant RR field' as a result of the BSFT.

\section{Acknowledgments}

The author would like to thank M.R.Garousi, A.Ghodsi, P.Vanhove and I.Y.Park for helpful conversations. He would also like to thank J.Polchinski for comments and A.Fotopoulos for useful suggestions during the winter school at CERN. It is a great pleasure to thank Luis Álvarez-Gaumé for several valuable discussions. The author also acknowledges the theory division of CERN for its hospitality. This work was supported by the Ministry of Science, Research and Technology in Iran.

Open Access. This article is distributed under the terms of the Creative Commons Attribution Noncommercial License which permits any noncommercial use, distribution, and reproduction in any medium, provided the original author(s) and source are credited.

\section{References}

[1] J. Polchinski, Dirichlet-Branes and Ramond-Ramond Charges, Phys. Rev. Lett. 75 (1995) 4724 [hep-th/9510017] [SPIRES].

[2] E. Witten, Bound states of strings and p-branes, Nucl. Phys. B 460 (1996) 335 [hep-th/9510135] [SPIRES].

[3] J. Polchinski, Lectures on D-branes, hep-th/9611050 [SPIRES].

[4] C.P. Bachas, Lectures on D-branes, hep-th/9806199 [SPIRES].

[5] P. Hořava, Type IIA D-branes, k-theory and matrix theory, Adv. Theor. Math. Phys. 2 (1999) 1373 [hep-th/9812135] [SPIRES].

[6] E. Witten, D-branes and k-theory, JHEP 12 (1998) 019 [hep-th/9810188] [SPIRES]. 
[7] J. Dai, R.G. Leigh and J. Polchinski, "New Connections Between String Theories," Mod. Phys. Lett. A4 (1989) 2073.

[8] A. Abouelsaood, C.G. Callan, Jr., C.R. Nappi and S.A. Yost, Open Strings in Background Gauge Fields, Nucl. Phys. B 280 (1987) 599 [SPIRES].

[9] C.G. Callan, C. Lovelace, C.R. Nappi and S.A. Yost, Loop Corrections to Superstring Equations of Motion, Nucl. Phys. B 308 (1988) 221 [SPIRES].

[10] R.G. Leigh, Dirac- Born-Infeld Action from Dirichlet $\sigma$-model, Mod. Phys. Lett. A 4 (1989) 2767 [SPIRES].

[11] A.A. Tseytlin, Born-Infeld action, supersymmetry and string theory, hep-th/9908105 [SPIRES].

[12] R.C. Myers, Dielectric-branes, JHEP 12 (1999) 022 [hep-th/9910053] [SPIRES].

[13] W. Taylor and M. Van Raamsdonk, Multiple Dp-branes in weak background fields, Nucl. Phys. B 573 (2000) 703 [hep-th/9910052] [SPIRES].

[14] M. Li, Boundary States of D-branes and Dy-Strings, Nucl. Phys. B 460 (1996) 351 [hep-th/9510161] [SPIRES].

[15] M.R. Douglas, Branes within branes, hep-th/9512077 [SPIRES].

[16] M.B. Green, J.A. Harvey and G.W. Moore, I-brane inflow and anomalous couplings on D-branes, Class. Quant. Grav. 14 (1997) 47 [hep-th/9605033] [SPIRES].

[17] P. Kraus and F. Larsen, Boundary string field theory of the DD-bar system, Phys. Rev. D 63 (2001) 106004 [hep-th/0012198] [SPIRES].

[18] T. Takayanagi, S. Terashima and T. Uesugi, Brane-antibrane action from boundary string field theory, JHEP 03 (2001) 019 [hep-th/0012210] [SPIRES].

[19] J. Polchinski, Combinatorics of boundaries in string theory, Phys. Rev. D 50 (1994) 6041 [hep-th/9407031] [SPIRES].

[20] J. Polchinski, S. Chaudhuri and C.V. Johnson, Notes on D-branes, hep-th/9602052 [SPIRES].

[21] I.Y. Park, Scattering on D3-branes, Phys. Lett. B 660 (2008) 583 [arXiv:0708.3452] [SPIRES].

[22] I.Y. Park, Open string engineering of D-brane geometry, JHEP 08 (2008) 026 [arXiv: 0806.3330] [SPIRES].

[23] A. Hashimoto and I.R. Klebanov, Scattering of strings from D-branes, Nucl. Phys. Proc. Suppl. 55B (1997) 118 [hep-th/9611214] [SPIRES].

[24] A. Hashimoto and I.R. Klebanov, Decay of Excited D-branes, Phys. Lett. B 381 (1996) 437 [hep-th/9604065] [SPIRES].

[25] M.R. Garousi and R.C. Myers, World-volume interactions on D-branes, Nucl. Phys. B 542 (1999) 73 [hep-th/9809100] [SPIRES].

[26] I.R. Klebanov and L. Thorlacius, The Size of p-Branes, Phys. Lett. B 371 (1996) 51 [hep-th/9510200] [SPIRES].

[27] S.S. Gubser, A. Hashimoto, I.R. Klebanov and J.M. Maldacena, Gravitational lensing by p-branes, Nucl. Phys. B 472 (1996) 231 [hep-th/9601057] [SPIRES].

[28] P.-J. De Smet and J. Raeymaekers, The tachyon potential in Witten's superstring field theory, JHEP 08 (2000) 020 [hep-th/0004112] [SPIRES]. 
[29] V. Balasubramanian and I.R. Klebanov, Some Aspects of Massive World-Brane Dynamics, Mod. Phys. Lett. A 11 (1996) 2271 [hep-th/9605174] [SPIRES].

[30] C. Bachas, D-brane dynamics, Phys. Lett. B 374 (1996) 37 [hep-th/9511043] [SPIRES].

[31] A. Sen, Non-BPS states and branes in string theory, hep-th/9904207 [SPIRES].

[32] G. Lifschytz, Comparing D-branes to Black-branes, Phys. Lett. B 388 (1996) 720 [hep-th/9604156] [SPIRES].

[33] J. Polchinski, String duality: A colloquium, Rev. Mod. Phys. 68 (1996) 1245 [hep-th/9607050] [SPIRES].

[34] W. Taylor, Lectures on D-branes, gauge theory and M(atrices), hep-th/9801182 [SPIRES].

[35] C. Vafa, Lectures on strings and dualities, hep-th/9702201 [SPIRES].

[36] M. Billó, M. Frau, F. Lonegro and A. Lerda, $N=1 / 2$ quiver gauge theories from open strings with RR fluxes, JHEP 05 (2005) 047 [hep-th/0502084] [SPIRES].

[37] M. Billó et al., Microscopic string analysis of the D0-D8 brane system and dual RR states, Nucl. Phys. B 526 (1998) 199 [hep-th/9802088] [SPIRES].

[38] M.R. Garousi and H. Golchin, On higher derivative corrections of the tachyon action, Nucl. Phys. B 800 (2008) 547 [arXiv:0801.3358] [SPIRES].

[39] N. Berkovits, SuperPoincaré invariant superstring field theory, Nucl. Phys. B 450 (1995) 90 [hep-th/9503099] [SPIRES].

[40] C. Kennedy and A. Wilkins, Ramond-Ramond couplings on brane-antibrane systems, Phys. Lett. B 464 (1999) 206 [hep-th/9905195] [SPIRES].

[41] R. Medina, F.T. Brandt and F.R. Machado, The open superstring 5-point amplitude revisited, JHEP 07 (2002) 071 [hep-th/0208121] [SPIRES].

[42] M.R. Garousi and E. Hatefi, On Wess-Zumino terms of Brane-Antibrane systems, Nucl. Phys. B 800 (2008) 502 [arXiv:0710.5875] [SPIRES].

[43] M.R. Garousi and E. Hatefi, More on WZ action of non-BPS branes, JHEP 03 (2009) 008 [arXiv:0812.4216] [SPIRES].

[44] S. Stieberger, Open, Closed vs. Pure Open String Disk Amplitudes, arXiv:0907.2211 [SPIRES].

[45] M.R. Garousi and R.C. Myers, Superstring Scattering from D-branes, Nucl. Phys. B 475 (1996) 193 [hep-th/9603194] [SPIRES].

[46] H. Liu and J. Michelson, *-trek III: The search for Ramond-Ramond couplings, Nucl. Phys. B 614 (2001) 330 [hep-th/0107172] [SPIRES].

[47] V.A. Kostelecky, O. Lechtenfeld, W. Lerche, S. Samuel and S. Watamura, Conformal Techniques, Bosonization and Tree Level String Amplitudes, Nucl. Phys. B 288 (1987) 173 [SPIRES].

[48] A. Fotopoulos, On $\left(\alpha^{\prime}\right)^{* * 2}$ corrections to the D-brane action for non- geodesic world-volume embeddings, JHEP 09 (2001) 005 [hep-th/0104146] [SPIRES].

[49] M.R. Garousi, Higher derivative corrections to Wess-Zumino action of Brane-Antibrane systems, JHEP 02 (2008) 109 [arXiv:0712.1954] [SPIRES].

[50] A. Neveu and J. Scherk, Connection between Yang-Mills fields and dual models, Nucl. Phys. B 36 (1972) 155 [SPIRES]. 
[51] J. Scherk and J.H. Schwarz, Dual Models For Nonhadrons, Nucl. Phys. B 81 (1974) 118 [SPIRES].

[52] A.A. Tseytlin, Vector Field Effective Action In The Open Superstring Theory, Nucl. Phys. B 276 (1986) 391 [Erratum-ibid. B 291 (1987) 876] [SPIRES].

[53] E.S. Fradkin and A.A. Tseytlin, Nonlinear Electrodynamics From Quantized Strings, Phys. Lett. B 163 (1985) 123 [SPIRES].

[54] E. Bergshoeff, E. Sezgin, C.N. Pope and P.K. Townsend, The Born-Infeld Action From Conformal Invariance Of The Open Superstring, Phys.Lett. B 188 (1987) 70 [SPIRES].

[55] R.R. Metsaev, M.A. Rahmanov and A.A. Tseytlin, The Born-Infeld Action As The Effective Action In The Open Superstring Theory, Phys. Lett. B 193 (1987) 207 [SPIRES].

[56] A.A. Tseytlin, Renormalization of Mobius Infinities and Partition Function Representation for String Theory Effective Action, Phys. Lett. B 202 (1988) 81 [SPIRES].

[57] A.A. Tseytlin, On non-abelian generalisation of the Born-Infeld action in string theory, Nucl. Phys. B 501 (1997) 41 [hep-th/9701125] [SPIRES].

[58] D. Gross and E. Witten, Superstring Modifications Of Einstein's Equations, Nucl. Phys. B 277 (1986) 1 [SPIRES].

[59] F. Denef, A. Sevrin and W. Troost, Non-Abelian Born-Infeld versus string theory, Nucl. Phys. B 581 (2000) 135 [hep-th/0002180] [SPIRES].

[60] A. Hashimoto and W. Taylor, Fluctuation spectra of tilted and intersecting D-branes from the Born-Infeld action, Nucl. Phys. B 503 (1997) 193 [hep-th/9703217] [SPIRES].

[61] P. Bain, On the non-Abelian Born-Infeld action, hep-th/9909154 [SPIRES].

[62] L. Cornalba, D-brane physics and noncommutative Yang-Mills theory, Adv. Theor. Math. Phys. 4 (2000) 271 [hep-th/9909081] [SPIRES].

[63] L. Cornalba, Corrections to the Abelian Born-Infeld action arising from noncommutative geometry, JHEP 09 (2000) 017 [hep-th/9912293] [SPIRES].

[64] Y. Okawa, Derivative corrections to Dirac- Born-Infeld Lagrangian and non-commutative gauge theory, Nucl. Phys. B 566 (2000) 348 [hep-th/9909132] [SPIRES].

[65] K. Bitaghsir-Fadafan and M.R. Garousi, Non-abelian expansion of S-matrix elements and non-abelian tachyon DBI action, Nucl. Phys. B 760 (2007) 197 [hep-th/0607249] [SPIRES].

[66] Y. Kitazawa, Effective Lagrangian for Open Superstring From Five Point Function, Nucl. Phys. B 289 (1987) 599 [SPIRES].

[67] M. Green, J. Schwarz and E. Witten, Superstring theory, Vol.1, Cambridge University Press, Cambridge U.K. (1987).

[68] J. Polchinski, String theory, Vol.1, Cambridge University Press, Cambridge U.K. (1998).

[69] A. Bilal, Higher-derivative corrections to the non-abelian Born-Infeld action, Nucl. Phys. B 618 (2001) 21 [hep-th/0106062] [SPIRES].

[70] O. Chandía and R. Medina, 4-point effective actions in open and closed superstring theory, JHEP 11 (2003) 003 [hep-th/0310015] [SPIRES].

[71] L.A. Barreiro and R. Medina, 5-field terms in the open superstring effective action, JHEP 03 (2005) 055 [hep-th/0503182] [SPIRES]. 